\title{
First report of segmented filamentous bacteria associated with Rhigonema sp. (Nematoda: Rhigonematidae) dwelling in hindgut of Riukiaria sp. (Diplopoda: Xystodesmidae)
}

\author{
Y. KITAGAMI ${ }^{1, *}$, N. KANZAKI ${ }^{2}$, Y. MATSUDA ${ }^{1}$ \\ ${ }^{1}$ Laboratory of Forest Mycology, Graduate School of Bioresources, Mie University, 1577 Kurimamachiya, Tsu, Mie 514-8507, Japan, \\ *E-mail: yudaikitagami@gmail.com; ${ }^{2}$ Kansai Research Center, Forestry and Forest Products Research Institute, \\ Nagai-kyutaro, Momoyama, Fushimi, Kyoto 612-0855, Japan
}

Article info

Received February 28, 2019

Accepted April 17, 2019

\section{Summary}

We morphologically and molecularly characterized segmented filamentous bacteria (SFB) associated with Rhigonema sp. nematodes in millipede hindguts. Seventy-three Riukiaria sp. millipedes were collected from a broad-leaf forest in Japan, and nematodes were excised from the millipede's hindguts. The occurrence rate of SFB associated with nematodes was $24 \%(10 / 41)$ for males, $47 \%$ (14/30) for females, and $100 \%$ (2/2) for juveniles. Genomic DNA was extracted from four SFB-rich nematode heads, and we obtained 40 bacterial clones via analysis of nearly full-length $16 \mathrm{~S}$ rDNA gene sequences. At the phylum level, Firmicutes, Proteobacteria, and Verrucomicrobia accounted for $55 \%, 40 \%$, and $5 \%$ of SFB, respectively. In Firmicutes, Clostridiaceae ( $28 \%$ ) and Lachnospiraceae (15\%) were the dominant groups. Our sequences were divided into seven and three subclades between Firmicutes and Proteobacteria in the phylogenetic tree. In the Firmicutes clade, eight sequences were classified as Lachnospiraceae with a bootstrap value $>83 \%$. A phylogenetic tree involving known uncultured Lachnospiraceae sequences characterized the phylogenetic position of SFB associated with nematodes. Our results suggest that the association of SFB with nematode bodies was probably incidental and that SFB are not always present in millipede hindguts. Our bacterial groups corresponded to those of arthropod hindgut, and SFB associated with nematodes were inferred to belong to Lachnospiraceae. Because the Lachnospiraceae sequences obtained in this study showed specific lineages that differed from all the known deposited sequence data, these groups may be unique to Riukiaria sp.

Keywords: Firmicutes; Lachnospiraceae; phylogenetic analysis; morphology; 16S rDNA

\section{Introduction}

Guts of many arthropods (e.g., termites, cockroaches, and millipedes) are inhabited by segmented filamentous bacteria (SFB), which are also referred to as "long segmented filamentous structures" (Margulis et al., 1998; Thompson et al., 2012). In the guts, these Gram-positive endospore-forming microbes are attached to the epithelial walls (Klaasen et al., 1992). Although SFB are difficult to culture in vitro, this group is well known as common constituents of gut microbes (Krecek et al., 1987; Brune \& Dietrich, 2015). Moreover, SFB were suggested to play a crucial role in host immune function through the coordination of T-cell responses (Ivanov et al., 2009).

Among soil-dwelling arthropods, nematodes have frequently been isolated from the guts of termites (Carta \& Osbrink, 2005), cockroaches (Ozawa et al., 2014), and millipedes (Hunt \& Moore, 1995; Morffe \& Hasegawa, 2017). SFB-like organisms were reported to be associated with the gut parasite nematodes Rhigonema spp.

\footnotetext{
$\bar{*}$ - corresponding author
} 
Table 1. Numbers of millipede Riukiaria sp., occurrence frequency of nematode Rhigonema sp., and segmented filamentous bacteria (SFB) associated with nematodes in millipede hindguts.

\begin{tabular}{lcccccccc}
\hline Sex & $\begin{array}{c}\text { Observed } \\
\text { numbers }\end{array}$ & $\begin{array}{c}\text { Body length } \\
(\mathrm{mm})\end{array}$ & \multicolumn{5}{c}{ Occurrence frequency of nematodes $^{\mathrm{b}}$} & \begin{tabular}{c} 
SFB associated \\
\cline { 5 - 7 }
\end{tabular} \\
\cline { 5 - 7 } with nematodes (\%)
\end{tabular}

${ }^{a}$ Data shown are mean $\pm \mathrm{SE}$.

${ }^{b}$ Numbers in parentheses indicate relative abundance. The frequency distribution of nematode abundances was significantly different between male and female millipedes ( $X^{2}$-test, $\left.X=21.164, \mathrm{df}=4, P<0.001\right)$, but there was no significant difference between the sexes with regard to occurrence rate of SFB associated with nematodes ( $X^{2}$-test, $\left.X=2.911, \mathrm{df}=1, P=0.08\right)$. Data on juveniles were not used in these analyses owing to the small sample size.

c Frequency of nematode individuals at each stage $(\%)=$ (number of nematodes in a certain range) $/$ (all nematodes observed in hindguts of millipedes) $\times 100$

${ }^{d}$ SFB associated with nematode at each stage $(\%)=$ (occurrence frequency of SFB attached nematodes) / (observed hindguts of millipedes) $\times 100$.

in millipede hindgut as early as the 19th century (Leidy, 1853). The SFB associated with Rhigonema spp. were also confirmed in hindguts of Riukiaria spp. (Xystodesmidae) in Japan (Kanzaki et al., 2016). However, the frequency of occurrence and phylogenetic position of SFB associated with Rhigonema sp. in millipede hindguts has not been investigated in detail.

The purpose of this study was to clarify the occurrence rate and phylogenetic position of SFB associated with nematodes. For this, we collected millipedes in a broad-leaf forest and characterized SFB associated with hindgut-dwelling nematodes by both morphological and molecular approaches.

\section{Materials and Methods}

Adults and juveniles of Riukiaria sp. were collected at Hinokuma Park in Saga Prefecture ( $\left.34^{\circ} 33^{\prime} \mathrm{N}, 130^{\circ} 35^{\prime} \mathrm{E}\right)$, Japan, in June 2018 (Fig. 1a). At the site, Cinnamomum camphora, Quercus aliena, Quercus serrata, and Schoepfia jasminodora of various ages were patchily distributed, and their broad-leaf litter covered the ground
(Fig. 1b). Annual precipitation and annual mean temperature in June 2018, as recorded at the nearest weather station in Saga (8 $\mathrm{km}$ from the site), were $291 \mathrm{~mm}$ and $24.1^{\circ} \mathrm{C}$, respectively.

The millipedes were brought back to the laboratory alive and stored at $20{ }^{\circ} \mathrm{C}$ for less than a month, until extraction of nematodes. The hypopygium of Riukiaria sp. was dissected with fine tweezers, and the hindgut was pulled out and excised. Rhigonema $\mathrm{sp}$. individuals were isolated from the hindgut of juvenile, male, and female millipedes in distilled water. All the Rhigonema nematodes retrieved from each millipede's hindgut were transferred into a Petri dish filled with distilled water. For each millipede, the numbers of Rhigonema sp. with and without SFB were counted under a stereomicroscope (SZX16, Olympus, Tokyo, Japan; maximum magnification $\times 115$ ).

We performed a $X^{2}$-test to ascertain whether the frequencies of occurrence of nematodes and SFB associated with nematodes in guts differed significantly between male and female millipedes. We also performed a Pearson's correlation test to assess the relationship between the number of nematodes per millipede and

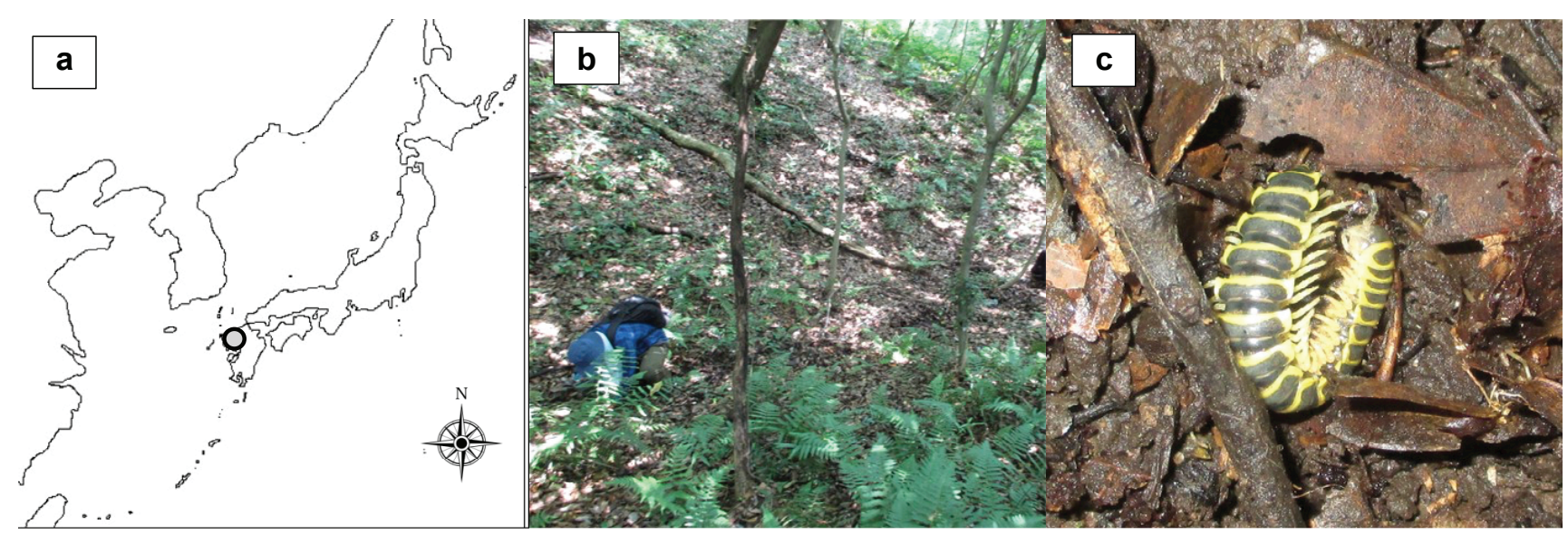

Fig. 1. Location of sampling site in Japan and a collected millipede. (a) The gray circle indicates the sampling location. Bar $=100 \mathrm{~km}$. (b) View of a sampled broad-leaf forest stand. (c) Riukiaria sp. collected beneath the litter layer on the ground. 
the number of nematodes associated with SFB. All data analyses were performed in R 3.3.2 ( $R$ Core Team, 2016), and the significance level was set at $P<0.05$.

Extracted nematodes were observed under a light microscope (BX53, Olympus; $\times 100-400$ ) for detailed observation of SFB. The nematodes were Gram stained with crystal violet (Wako, Osaka, Japan) for the presence of SFB, then photographed (TIFF format, $1360 \times 1024$ pixels) with a digital camera (DP70, Olympus) connected to the microscope.

Genomic DNA was extracted from four SFB-rich nematode heads using the DNeasy Plant Mini Kit (Qiagen, Valencia, CA, USA). To obtain nearly the full length of $16 \mathrm{~S}$ rDNA, the extracted DNAs were amplified using Tks Gflex (Takara, Shiga, Japan) with the primer pair 9f (GAGTTTGATCCTGGCTCAG; Yoon et al., 1997) and 1541r (AAGGAGGTGATCCAGCCG; Sato et al., 2004). Thermal conditions were one cycle of $94^{\circ} \mathrm{C}$ for $1 \mathrm{~min}$, followed by 30 cycles of $98^{\circ} \mathrm{C}$ for $10 \mathrm{sec}, 55^{\circ} \mathrm{C}$ for $30 \mathrm{sec}$, and $72{ }^{\circ} \mathrm{C}$ for $90 \mathrm{sec}$. Positive $\mathrm{PCR}$ products were cloned using the TA-Enhancer Cloning Kit (Nippon Gene, Toyama, Japan) according to the manufacturer's instructions. Sixteen successfully inserted white colonies were picked up per nematode head part. Selected colonies were further amplified with Tks Gflex and the primer pair 9f and 1541r. The ther- mal cycle program comprised 25 cycles of $98^{\circ} \mathrm{C}$ for $10 \mathrm{sec}, 55^{\circ} \mathrm{C}$ for $30 \mathrm{sec}$, and $72.0^{\circ} \mathrm{C}$ for $90 \mathrm{sec}$. When PCR amplicons were successfully produced, they were purified by using ExoSAP-IT PCR Product Cleanup Reagent (Affymetrix, Thermo Fisher Scientific, Waltham, MA, USA) in accordance with the manufacturer's instructions. Purified DNAs were labeled by using a BigDye Terminator v3.1 Cycle Sequencing Kit (Applied Biosystems, Carlsbad, CA, USA) and then sequenced by using an $A B I 3730$ sequencer (Applied Biosystems) with one of the three primers - 9f, 1541r, or $533 f$ (GTGCCAGCAGCCGCGGTAA; Weisburg et al., 1991) - to obtain full-length $16 \mathrm{~S}$ rDNAs.

Obtained sequences were adjusted manually in MEGA v. 7 software (Kumar et al., 2016; https://www.megasoftware.net/), and sequences of the same sample read by different primers were assembled into a longer sequence whenever possible. Forty sequences were deposited in the DDBJ under accession numbers LC462722-LC462761. Sequences were compared with deposited sequences and identified to the nearest taxon using pairwise searches with the basic local alignment search tool (BLAST) (Altschul et al., 1997; https://blast.ncbi.nlm.nih.gov/Blast.cgi).

To estimate the phylogenetic positions of the bacteria, we constructed phylogenetic trees by using the maximum likelihood
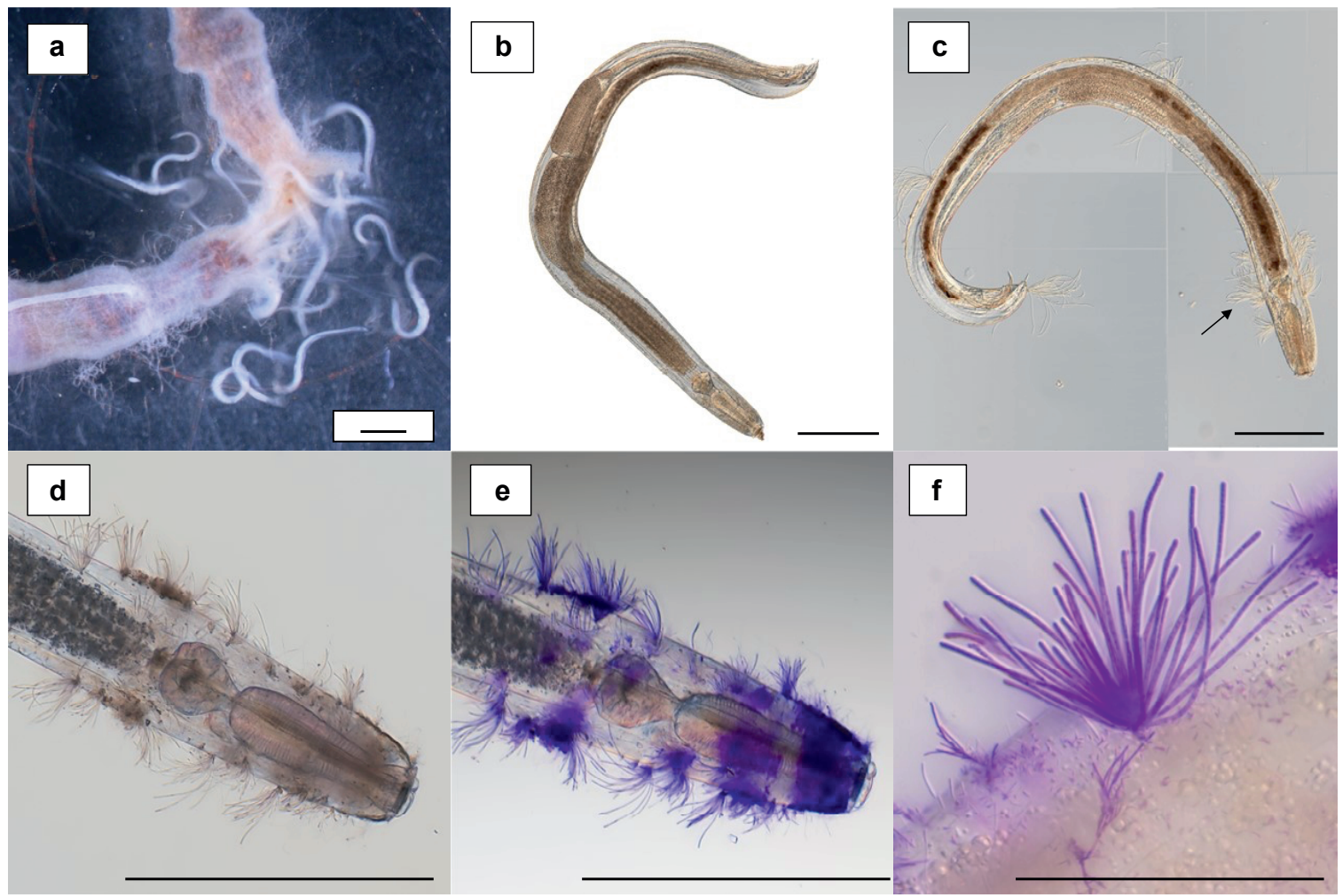

Fig. 2. Micrographs of Rhigonema sp. isolated from millipede guts. Images show whole individual and enlarged head part. (a) Rhigonema sp. crawl out from a millipede hindgut. (b) Rhigonema sp. without segmented filamentous bacteria (SFB). (c) Rhigonema sp. with SFB (arrow). (d) Enlarged head part of Rhigonema sp. with associated SFB. (e) Gram staining with crystal violet of associated SFB at head part. (f) SFB attached to nematode body surface. Bars represent (a) $1 \mathrm{~mm}$, (b) $500 \mu \mathrm{m}$, and (f) $100 \mu \mathrm{m}$. 


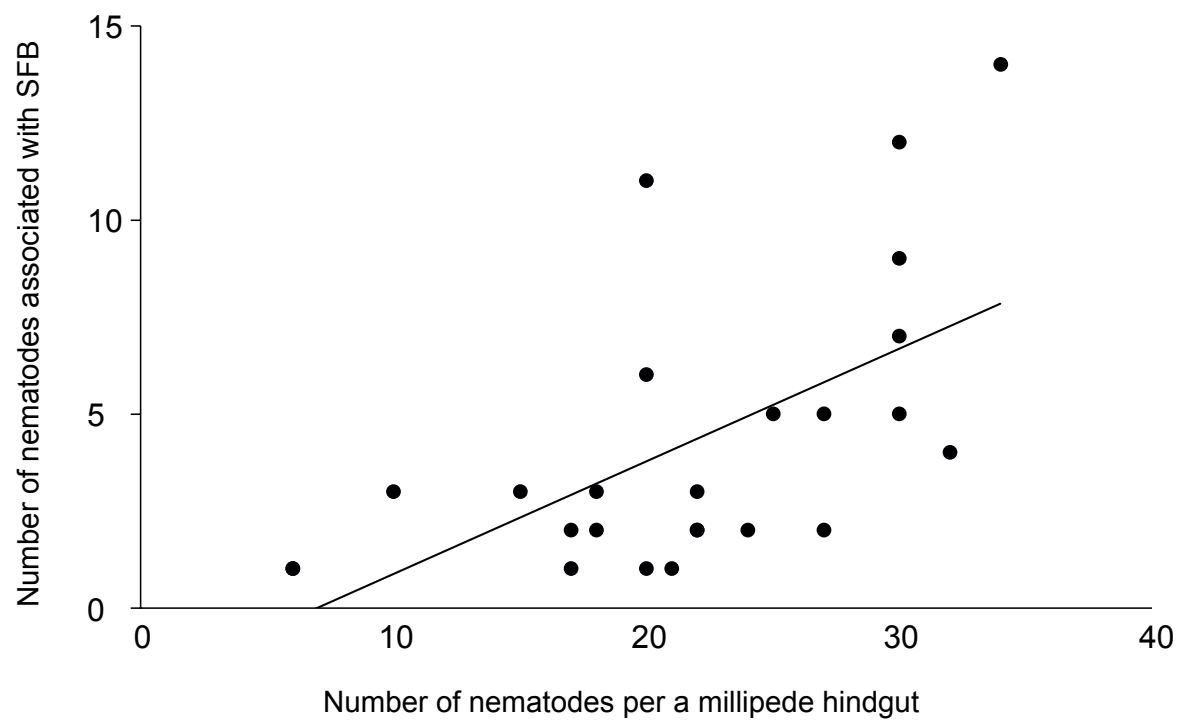

Fig. 3. Correlation between number of Rhigonema sp. nematodes per Riukiaria sp. millipede hindgut and number of nematodes associated with segmented filamentous bacteria (SFB), for those millipedes that had at least one nematode associated with SFB $(n=25$, Pearson's correlation test, $r=0.593, P=0.0017)$.

method in MEGA. For the default substitution model, we selected General Time Reversible (GTR) with Gamma-distributed rates plus Invariant sites $(\mathrm{G}+\mathrm{I})$, which had the smallest Akaike's information criterion (AIC) and Bayesian information criterion (BIC) based on the model selection implemented in MEGA. To draw the trees, we referred to the clone library of bacterial sequences derived from the cockroach Shefordella lateralis (Schauer et al., 2012), the termite Reticulitermes santonensis, and the millipede Tachypodoiulus niger (Thompson et al., 2012) deposited in GenBank. The sequences were aligned in MAFFT v. 7 software (Katoh \& Standley, 2013; http://mafft.cbrc.jp/alignment/software/) with default settings. In addition, we used the closest sequences of named family

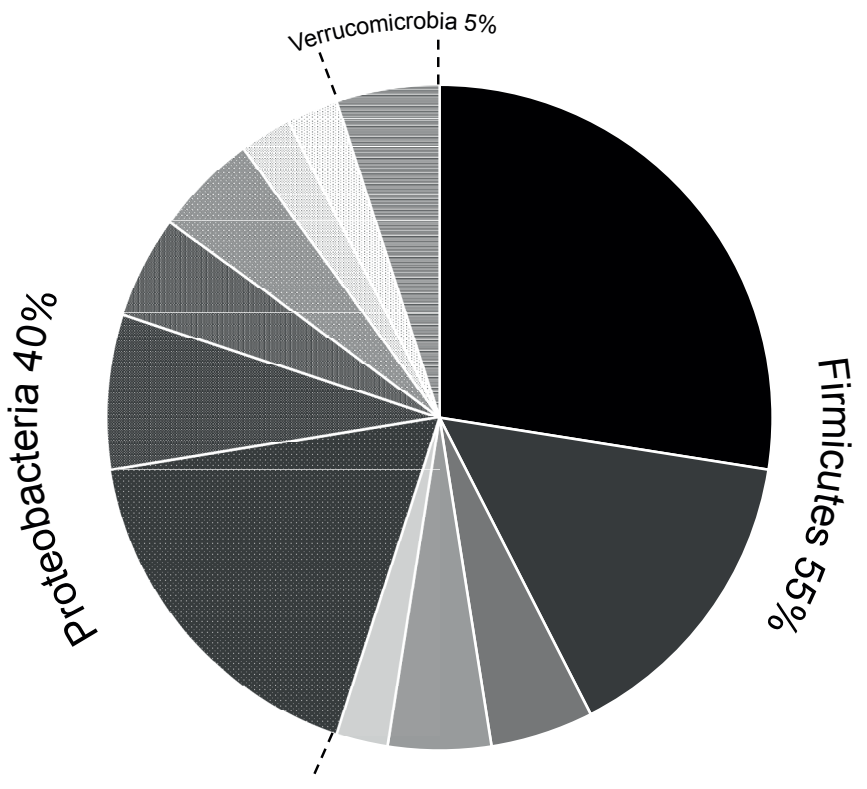

Clostridiaceae

- Lachnospiraceae

- Eubacteriaceae

numinococcaceae

- Lysinobacillus sp.

- Desulfovibrionaceae

Denitratisoma sp.

䊫 Cystobacteraceae

Janthinobacterium sp.

Propionivibrio sp.

Shigella sp.

豆 Verrucomicrobia

Fig. 4. Occurrence rates (\%) of bacterial taxa detected from segmented filamentous bacteria-enriched Rhigonema sp. heads in hindgut of Riukiaria sp. (40 clones). 


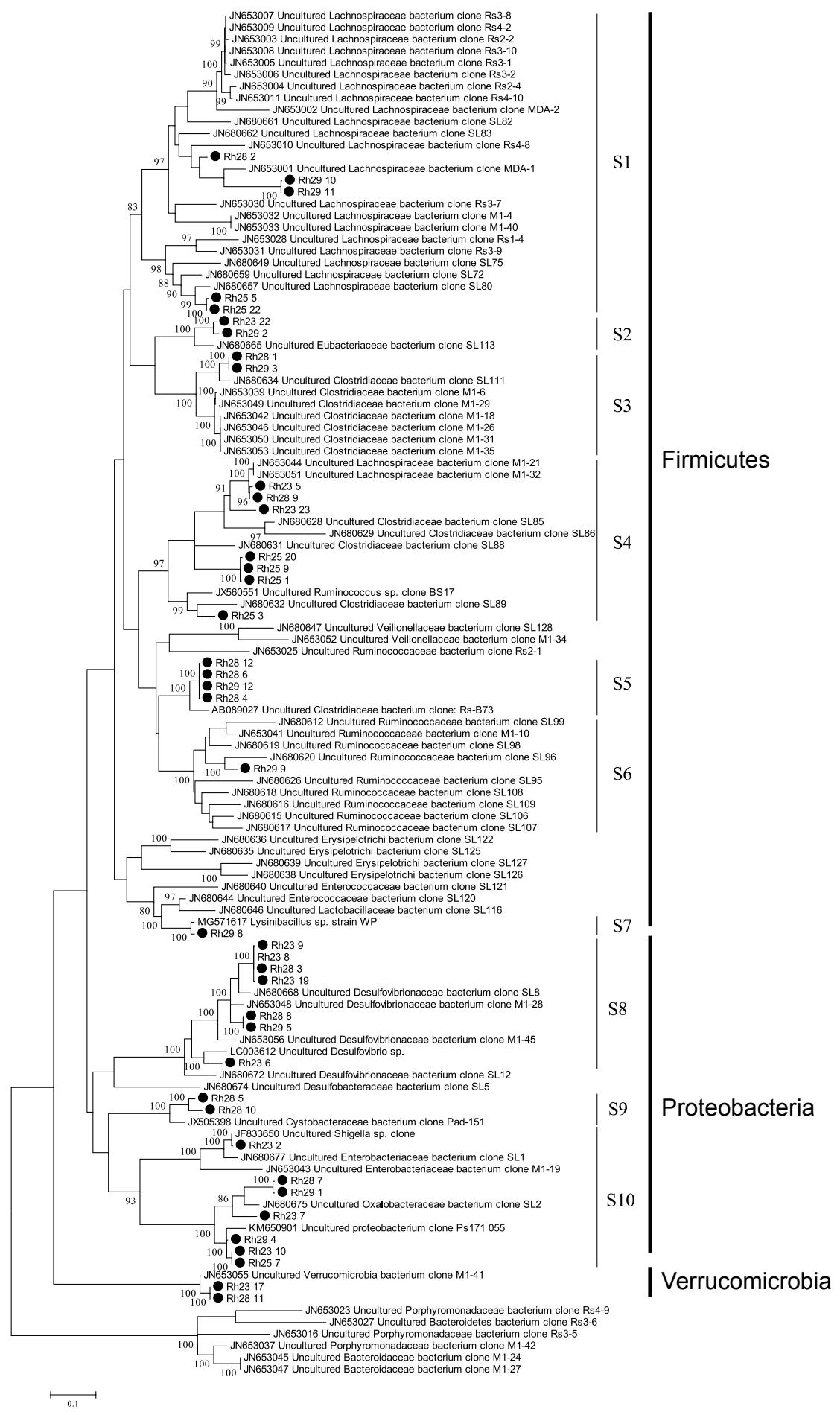

Fig. 5. A maximum likelihood phylogenetic tree of segmented filamentous bacteria (SFB) associated with Rhigonema sp. based on partial $16 \mathrm{~S}$ rDNA sequences. The substitution model was GTR with $\mathrm{G}+\mathrm{I}$ sites (AIC $=33224.486, \mathrm{BIC}=35454.324$ ). We used clone libraries of 76 bacterial sequences as ingroups derived from the cockroach Shefordella lateralis (Schauer et al., 2012), termite Reticulitermes santonensis, and millipede Tachypodoiulus niger (Thompson et al., 2012) deposited in GenBank. No outgroups were used. Values $\geq 80 \%$ on branches indicate confidence limits estimated by bootstrap analysis with 1000 replicates. 


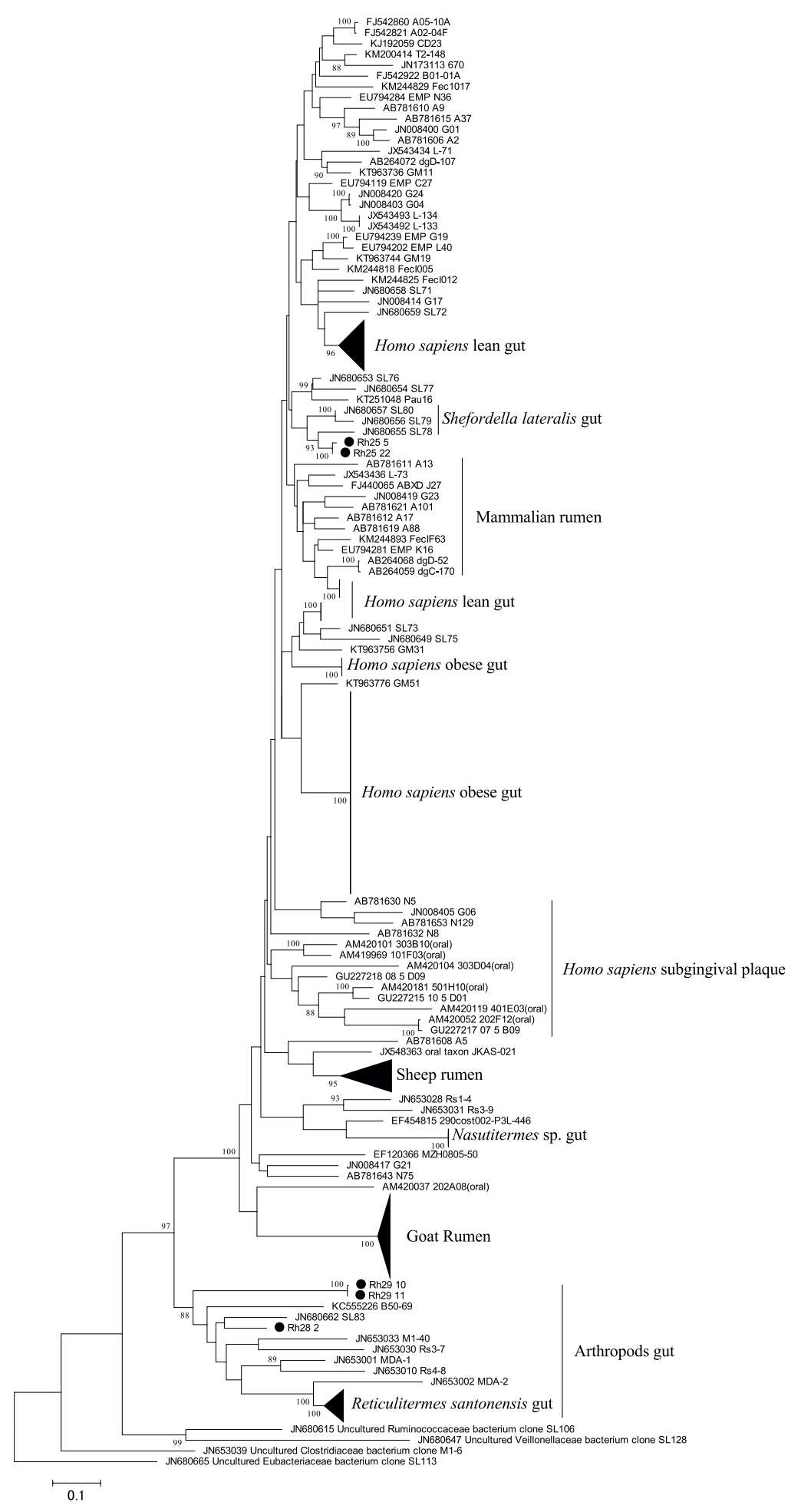

Fig. 6. A maximum likelihood phylogenetic tree of associated Lachnospiraceae groups of subclade S1 based on partial 16S rDNA sequences. The substitution model was GTR with $\mathrm{G}+\mathrm{I}$ sites $(\mathrm{AIC}=53520.724, \mathrm{BIC}=57505.700$ ). Known sequences of 181 uncultured Lachnospiraceae with nearly the full length of $16 \mathrm{~S}$ rDNA deposited in GenBank were used as ingroup sequences. Clostridiaceae (JN653039), Eubacteriaceae (JN680665), Ruminococcaceae (JN680615), and Veillonellaceae (JN680647) were used as outgroups. Values $\geq 80 \%$ on branches indicate confidence limits estimated by bootstrap analysis with 1000 replicates. 


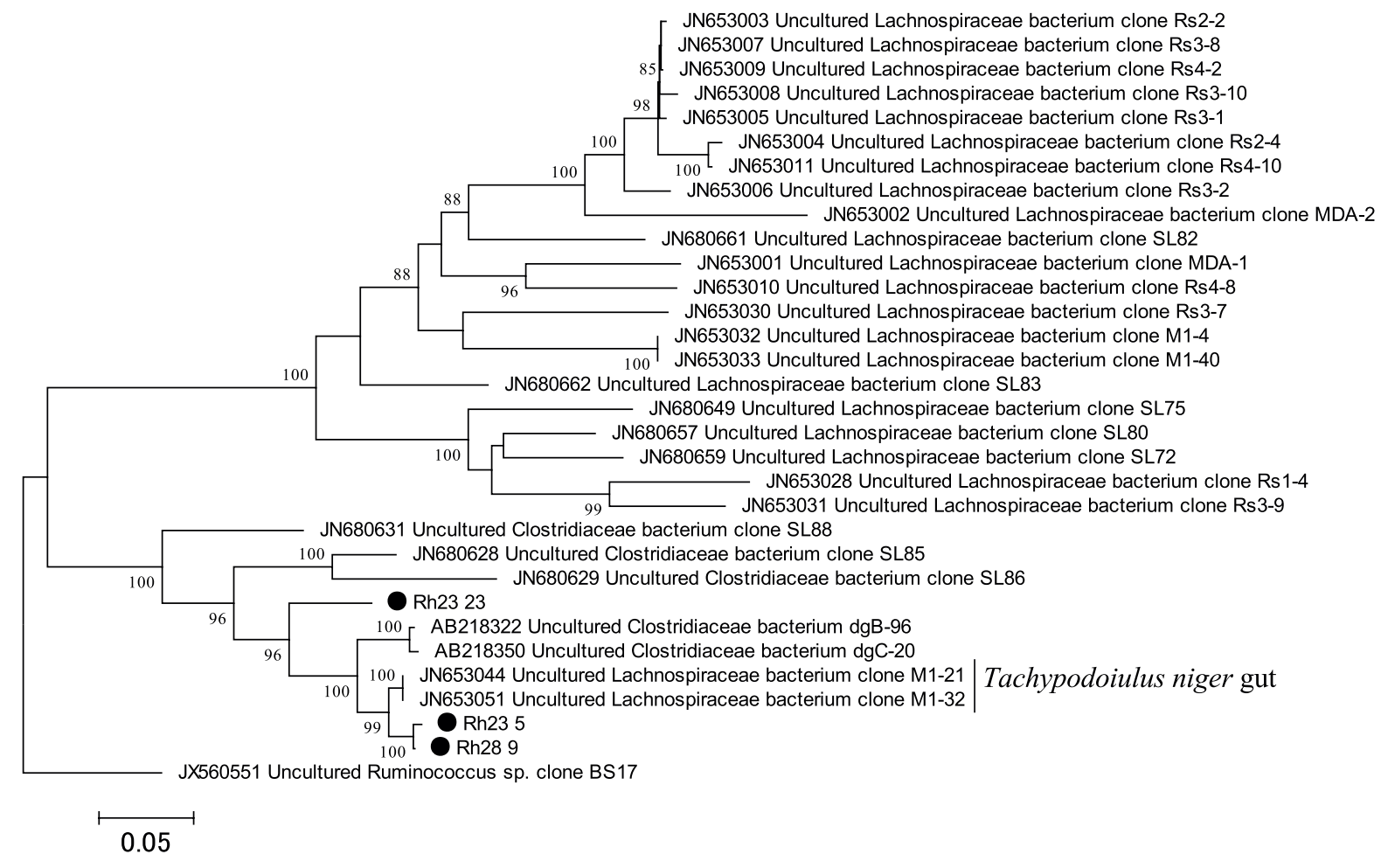

Fig. 7. A maximum likelihood phylogenetic tree of associated Lachnospiraceae groups of subclade S4 based on partial $16 \mathrm{~S}$ rDNA sequences. The substitution model was GTR with G+I sites (AIC $=20633.765, \mathrm{BIC}=21242.547$ ). As ingroup sequences, we used known sequences of 26 uncultured Lachnospiraceae with nearly the full length of $16 \mathrm{~S}$ rDNA derived from the cockroach Shefordella lateralis (Schauer et al., 2012), termite Reticulitermes santonensis, and millipede Tachypodoiulus niger (Thompson et al., 2012) deposited in GenBank. Ruminocccus sp. (JX560551) was used as the outgroup. Values $\geq 80 \%$ on branches indicate confidence limits estimated by bootstrap analysis with 1000 replicates.

or genus in GenBank identified by using pairwise BLAST searches as in-group sequences. To increase the resolution of phylogenetic positions, the sequences that were tentatively assigned to Lachnospiraceae (see Results) were re-analyzed by the maximum likelihood method as above involving all the known uncultured Lachnospiraceae sequences with nearly the full length of $16 \mathrm{~S}$ rDNA deposited in GenBank. For the default substitution model, we selected GTR with $\mathrm{G}+\mathrm{I}$, which had the smallest AIC and BIC based on the model selection in MEGA. For the Lachnospiraceae tree, Clostridiaceae (JN653039), Eubacteriaceae (JN680665), Ruminococcaceae (JN680615), and Veillonellaceae (JN680647) in subclade S1 (see Fig. 6), Ruminocccus sp. (JX560551 in subclade S4 (see Fig. 7) were used as outgroups. The reliability of all tree topologies was evaluated by 1000 bootstrap resamplings (Felsenstein, 1985).

\section{Ethical Approval and/or Informed Consent}

The research related to animals has been complied with all the relevant national regulations and institutional policies for the care and use of animals.

\section{Results}

The microscopy of Rhigonema sp. demonstrated that SFB attached to the nematode body (Fig. $2 a-d$ ), and the parts associated with SFB were clearly stained purple with the crystal violet (Fig. 2e, f). The occurrence rates of Rhigonema sp. in millipede hindguts were $90 \%(37 / 41)$ in males, $100 \%$ (30/30) in females, and $100 \%(2 / 2)$ in juveniles (Table 1). The highest frequency of Rhigonema sp. per hindgut was $41 \%$ for males harboring $1-10$ individuals and $64 \%$ for females harboring 21 - 30 individuals. The relative abundance of nematodes was significantly different between male and female millipedes (Table 1, $X^{2}$-test, $X=21.164$, $\mathrm{df}=4, P<0.001)$. The occurrence rate of SFB associated with nematodes was $24 \%$ (10/41) for males, $47 \%$ (14/30) for females, and $100 \%$ (2/2) for juveniles, and there was no significant difference between sexes (Table 1, $X^{2}$-test, $X=2.911$, df $=1, P=0.08$ ). We noted a significant positive correlation between the number of nematodes per millipede and the number of nematodes associated with SFB (Fig. 3, Pearson's correlation test, $n=25, r=0.593$, $P=0.0017)$.

Since 6 clones were identified as Escherichia coli (negative con- 
trol) by BLAST search, they were excluded. DNA sequences were obtained from $69 \%$ (40/58) of clones. At the phylum level, Firmicutes, Proteobacteria and Verrucomicrobia accounted for $55 \%$, $40 \%$, and $5 \%$, respectively (Fig. 4). In the Firmicutes, Clostridiaceae $(28 \%)$ and Lachnospiraceae $(15 \%)$ were the dominant groups, whereas Desulfovibrionaceae (18\%) dominated the Proteobacteria. These clones were used for the construction of a maximum likelihood tree with known, named sequences (Fig. 5). Our sequences were affiliated with taxonomic clades (Firmicutes, Proteobacteria, or Verrucomicrobia) in the tree. Among them, Firmicutes and Proteobacteria harbored seven and three subclades, respectively, with bootstrap (BS) values greater than $83 \%$ (Fig. 5). The subclade $\mathrm{S} 1$ formed with Lachnospiraceae lineages, and two of five sequences (Rh25_5, Rh25_22) were close to uncultured Lachnospiraceae derived from S. lateralis (JN680655), with a $93 \%$ BS value (Fig. 6). On the other hand, clones of Rh28_2, Rh29_10, and Rh29_11 formed a clade with an $88 \%$ BS value. In a tree retrieved from the $S 4$ clade, three of our sequences were positioned differently from most Lachnospiraceae sequences, with $100 \%$ BS values (Fig. 7). The tree was an admix lineage of Clostridiaceae and Lachnospiraceae, and three sequences (Rh23_5, Rh23_23, Rh28_9) were nested within Clostridiaceae groups neighboring Lachnospiraceae clones, with a $99 \%$ BS value.

\section{Discussion}

Our results clearly showed that most nematodes in millipede hindguts were associated with SFB. This study is the first to reveal the identity of SFB associated with nematodes by using molecular methods. Moreover, our bacterial sequences were clustered with known data derived from arthropod guts at high BS values. On the basis of these results, we discuss the occurrence frequency and phylogenetic position of SFB associated with nematodes.

Rhigonema spp. are assumed to be common in hindguts of Riukiaria spp. in Japan (Kanzaki et al., 2016), and the frequency of occurrence of the nematode in millipede guts was high in both sexes (Table 1). However, the number of nematodes per millipede gut was significantly greater in females than males. Females feed more actively than males in order to obtain more nutrients for oviposition (Boggs, 1981), so nutritional levels likely differ between sexes, as reflected in the population size of nematodes in their guts. The occurrence rate of SFB associated with nematodes was less than $50 \%$ for both sexes. Moreover, the number of nematodes per millipede hindgut and the number of nematodes associated with SFB were significantly and positively correlated. Thus, the SFB density in millipede guts might depend on the population size of parasitizing nematodes.

Microscopy of Rhigonema sp. revealed that associating SFB were Gram-positive, formed endospores, and attached to the body of nematodes. These morphological characters agree well with previous reports (Klaasen et al., 1992). Leidy (1853) observed a SFB associated with Rhigonema infectum, Thelastoma attenuatum and Aorurus agilis in the hindgut of the millipede Narceus annularis (summarized in Sayre \& Starr, 1988). Further, more recently, Blatta orientalis, Leidynema spp. nematodes were found to be covered with SFB in the hindgut of black cockroach, Blatta orientalis (Dr. Sergei Spiridonov, pers. comm.). Thus, SFB might generally associate with nematodes harbored within arthropod hindguts.

The most representative phyla in this study were Firmicutes and Proteobacteria, which were also dominant in the hindgut of Japanese cockroach, Periplaneta japonica (Vicente et al., 2018). The most frequent taxa were Clostridiaceae and Lachnospiraceae, which are anaerobic and unculturable taxa known to be an abundant group in arthropod guts (Engel \& Moran, 2013; Vicente et al., 2018). Our sequence data were nested with known anaerobic bacteria in gut conditions of millipedes, termites, and cockroaches with a high BS probability (>80 \% BS, Fig. 5). Common bacterial groups in arthropods' hindguts were attached to nematode head parts. The SFB in the hindgut of the termite $R$. santonensis and the millipede $T$. niger were classified phylogenetically to a group of Lachnospiraceae (Thompson et al., 2012). Lachnospiraceae were common in the hindgut of arthropods, such as termites, cockroaches, and millipedes, and were attached to their gut walls (Thompson et al., 2012). In the Lachnospiraceae tree in subclade S1, our sequences were clearly separated from other known sequences (100 \% BS, Fig. 6). Moreover, the refined tree of subclade S4 was constructed by admixed sequences of both Clostridiaceae and Lachnospiraceae; our sequences were nested within the former taxon but clearly differed from most members of the latter taxon (Fig. 7). The phylum Firmicutes included representatives of common gut bacteria, with most belonging to the family Lachnospiraceae, and highly specific lineages associated with the hindgut cuticle of arthropods (Brune \& Dietrich, 2015). Since the sequences obtained in this study also showed specific lineages differing from all the known deposited sequence data, these groups might be unique to Riukiaria sp. In addition, one of our obtained sequences was not placed with any known taxa, implying that the sequence is from an as-yet-unknown taxon. The Lachnospiraceae lineages were distributed among various locations and suggested to include cryptic species, indicating that the phylogenetic relationship within this group should be further investigated.

Our morphological and molecular analyses helped to characterize the SFB associated with nematodes within millipede hindguts in Japan. The association of SFB with nematode bodies was probably incidental and did not occur in all millipede hindguts. While the function of SFB in arthropod guts remains unknown, Ivanov et al. (2009) reported their crucial role in a host immune function through the coordination of T-cell responses. The distribution and functional significance of nematode-associated SFB remain unclear. To resolve these issues, in future research we plan to observe the ultrafine structure of SFB-associated parts in nematodes. 


\section{Acknowledgments}

This study was supported in part by Grants-in-Aid for Scientific Research (B) (No. 17H03831 to NK, 18H02237 to YM) and JSPS Research Fellowship 18J13285 to YK from the Japan Society for the Promotion of Science. The funder had no role in study design, data collection and analysis, decision to publish, or preparation of the manuscript. The collection of millipedes was permitted by the staff of Saga prefecture. We thank T. Chikada and members of the Life Science Research Center, Center for Molecular Biology and Genetics, Mie University, for helping with DNA analyses, and Dr. T. Ekino (Meiji University) for supporting our field sampling.

\section{Conflict of Interest}

The authors declare that there are no conflicts of interest.

\section{References}

Altschul, S.F., Madden, T.L., Schäffer, A.A., Zhang, J., Zhang, Z., Miller, W., LIPMAN, D.J. (1997): Gapped BLAST and PSI-BLAST: a new generation of protein database search programs. Nucleic Acids Res., 25: 3389 - 3402. DOI: 10.1093/nar/25.17.3389

Boggs, C. L. (1981): Nutritional and life-history determinants of resource allocation in holometabolous insects. Am. Nat., 117: 692 $-709$

Brune, A., Dietrich, C. (2015): The gut microbiota of termites: digesting the diversity in the light of ecology and evolution. Annu. Rev. Microbiol., 69: 145 - 166. DOI: 10.1146/annurev-micro-092412-155715

CARTA, L.K., OsBrinK, W. (2005): Rhabditis rainai n. sp. (Nematoda: Rhabditida) associated with the Formosan subterranean termite, Coptotermes formosanus (Isoptera: Rhinotermitidae). Nematology, 7: 863 - 879. DOI: 10.1163/156854105776186299

Engel, P., Moran, N.A. (2013): The gut microbiota of insects: diversity in structure and function. FEMS Microbiol. Rev. 37: 699 - 735. DOI: 10.1111/1574-6976.12025

FELsenstein, J. (1985): Confidence limits on phylogenies: an approach using the Bootstrap. Evolution, 39: 783 - 791. DOI: $10.2307 / 2408678$

Hunt, D.J., Moore, D. (1995): Rhigonema trichopeplum sp. n. (Nematoda: Rhigonematidae), parasite of a millipede (Diplopoda: Spirobolida) from Myanmar. Fundam. Appl. Nematol., 18: 553 558

Ivanov, I.I., Atarashi, K., Manel, N., Brodie, E.L., Shima, T., Karaoz, U., Wei, D., Goldfarb, K.C., Santee, C.A., Lynch, S. V., Tanoue, T., ImAOKA, A., Itoh, K., TAKedA, K., UmesaKI, Y., Honda, K., LitTMAn, D.R. (2009): Induction of intestinal Th17 cells by segmented filamentous bacteria. Cell, 139: 485 - 498. DOI: 10.1016/j.cell.2009.09.033 Kanzaki, N., Ekino, T., Tanaka, R., Woodruff, G.C., Ide, T., Yoshiga, T. (2016): Preliminary survey of millipede-associated nematodes in Japan: Nematode isolation from three Riukiaria spp. Nematol.
Res., 43: 105 - 106 (abstract for annual meeting of the Japanese Nematological Society)

KatoH, K., StandLEY, D.M. (2013): MAFFT multiple sequence alignment software version 7: Improvements in performance and usability. Mol. Biol. Evol., 30: 772 - 780. DOI: 10.1093/molbev/mst010 Klaasen, H.L.B.M., Koopman, J.P., Poelma, F.G.J., Beynen, A.C. (1992): Intestinal, segmented, filamentous bacteria. FEMS Microbiol. Lett., 88: 165 - 179. DOI: 10.1016/0378-1097(92)90801-T

Krecek, R.C., Sayre, R.M., Els, H.J., van Niekerk, J.P., Malan, F.S. (1987): Fine Structure of a Bacterial Community Associated with Cyathostomes (Nematoda: Strongylidae) of Zebras. Proc. Helminthol. Soc. Wash., 54: 212 - 219

Kumar, S., Stecher, G. Tamura, K. (2016): MEGA7: molecular evolutionary genetics analysis version 7.0 for bigger datasets. Mol. Biol. Evol., 33: 1870 - 1874. DOI: 10.1093/molbev/msw054

LEIDY, J. (1853): A Flora and Fauna within Living Animals. Smithsonian Institution, G. P. Putnam and Company, New York. 67 pp. Margulis, L., Jorgensen, J.Z., Dolan, S., Kolchinsky, R., Rainey, F.A., Lo, S.C. (1998): The Arthromitus stage of Bacillus cereus: intestinal symbionts of animals. Proc. Natl. Acad. Sci. USA. 95: 1236 - 1241. DOI: 10.1073/pnas.95.3.1236

Morffe, J., Hasegawa, K. (2017): Rhigonema naylae n. sp. (Rhigonematomorpha: Rhigonematidae) a new parasitic nematode from a Japanese polydesmid millipede (Polydesmida: Xystodesmidae). Zootaxa, 4269: 277 - 286. DOI: 10.11646/zootaxa.4269.2.6

Ozawa, S., Vicente, C.S.L., Sato, K., Yoshiga, T., Kanzaki, N., HasegAWA, K. (2014): First report of the nematode Leidynema appendiculata from Periplaneta fuliginosa. Acta Parasitol., 59: 219 - 228. DOI: 10.2478/s11686-014-0230-6

R Development Core Team (2016): R: a language and environment for statistical computing. $\mathrm{R}$ foundation for statistical computing, Austria. https://www.R-project.org/

Sato, Y., Nishihara, H., Yoshida, M.,Watanabe, M., Rondal, J.D., ОнтA, H. (2004): Occurrence of hydrogen-oxidizing Ralstonia species as primary microorganisms in the Mt. Pinatubo volcanic mudflow deposits. Soil Sci. Plant Nutr., 50: 855 - 861. DOI: 10.1080/ 00380768.2004 .10408546

SAYRE, R.M., StARR, M.P. (1988): Bacterial Disease and Antagonisms of Nematodes. In: Polnar, G.O., Jansson, H.B. (Eds) Disease of Nematodes. Volume 1. Boca Raton, FL: CRC Press, pp. $95-96$

Schauer, C., Thompson, C.L., Brune, A. (2012): The bacterial community in the gut of the cockroach Shelfordella lateralis reflects the close evolutionary relatedness of cockroaches and termites. Appl. Environ. Microbiol., 78: 2758 - 2767. DOI: 10.1128/AEM.07788-11 Thompson, C.L., Vier, R., Mikaelyan, A., Wienemann, T., Brune, A. (2012): 'Candidatus Arthromitus' revised: segmented filamentous bacteria in arthropod guts are members of Lachnospiraceae. Environ. Microbiol., 14: 1454 - 1465. DOI: 10.1111/j.14622920.2012.02731.x

Vicente, C. S. L., Ozawa, S., Hasegawa, K. (2018): The composi- 
tion of hindgut microbiota of Periplaneta japonica in the presence of thelastomatid parasitic nematodes. Nematol Res. 48: $19-26$. DOI: $10.3725 / j \mathrm{jn} .48 .19$

Weisburg, W. G., Barns, S. M., Pelletier, D. A., Lane, D. J. (1991): 16S ribosomal DNA amplification for phylogenetic study. J. Bacteriol. 173, 697 - 703. DOI: 10.1128/jb.173.2.697-703.1991
Yoon, J.H., Lee, S.T., Kim, S.B., Kim, W.Y., Goodfellow, M., Park, Y.H. (1997): Restriction fragment length polymorphism analysis of PCR-amplified 16S ribosomal DNA for rapid identification of Saccharomonospora strains. Int. J. Syst. Evol. Microbiol., 47: 111 114. DOI: $10.1099 / 00207713-47-1-111$ 